\title{
Orbital Anatomy: Anatomical Relationships of Surrounding Structures
}

\author{
Laura Salgado-López $^{1} \quad$ Luciano C.P. Campos-Leonel ${ }^{1}$ Carlos D. Pinheiro-Neto ${ }^{2}$ María Peris-Celda ${ }^{1}$ \\ ${ }^{1}$ Department of Neurosurgery, Albany Medical Center, Albany, New \\ York, United States \\ 2 Division of Otolaryngology and Head-Neck Surgery, Department of \\ Surgery, Albany Medical Center, Albany, New York, United States

\begin{abstract}
Address for correspondence Maria Peris-Celda, MD, PhD, Department of Neurosurgery, Albany Medical Center, 43 New Scotland Avenue, Albany, NY 12208, United States
\end{abstract} \\ (e-mail: periscm@amc.edu).
}

J Neurol Surg B 2020;81:333-347.

\begin{abstract}
Keywords

- ethmoid bone

- inferior orbital fissure

- maxillary sinus

- optic canal

- superior orbital fissure
\end{abstract}

\section{Introduction}

Advances in skull base and orbital surgery have led to an increased need for an understanding of the anatomy of the orbit and its relationship to the surrounding areas.

Tumors located in the anterior two-thirds of the orbit can often be approached anteriorly through the palpebrae and conjunctivae, and, depending on the location, can also be approached laterally through the lateral orbital wall, or superiorly through a transcranial approach. Retrobulbar lesions medial to the optic nerve are usually amenable to endoscopic endonasal approaches. Lesions located in the orbital apex, especially those on the medial side of the optic nerve, often require endonasal endoscopic approaches, whereas the lesions located lateral to the optic nerve are usually approached through a transcranial approach or lateral orbitotomy. ${ }^{1}$ A deep knowledge of the critical neurovascular and osseous structures in this region is necessary for adequately choosing and performing the most favorable orbital approach in every case.
The purpose of this article is to review the surrounding anatomy of the orbit from a practical operative point of view.

\section{Results}

\section{The Orbit from an Inferomedial Perspective: The Endoscopic Endonasal Approach}

Medial Relationships: The Ethmoid Bone

The endoscopic approach through the lamina papyracea has demonstrated many advantages as compared with the traditional medial orbitotomies for certain orbital lesions., 2,3 Endoscopic treatment of sinus diseases may be associated with serious orbital complications when the extremely thin or even dehiscent lamina papyracea is mistaken for ethmoid cells.

The medial orbital wall, orbital apex, and medial optic canal can be readily accessed through an endonasal approach. The medial orbital wall is composed of the frontal process of the maxilla, the lacrimal bone, the orbital plate of

(C) 2020 Georg Thieme Verlag KG Stuttgart · New York
DOI https://doi.org/ 10.1055/s-0040-1713931. ISSN 2193-6331. published online

August 24, 2020 


\section{Pearls and Tips}

- Before endoscopic endonasal approaches to the orbit, it is critical to study preoperative imaging studies to identify anatomical variants that may increase surgical complexity or increase the risk of neurovascular injury such as a pneumatized middle turbinate (concha bullosa), infraorbital ethmoid cell (Haller's cells), or sphenoethmoidal cell (Onodi's cell).

- The middle turbinate should be handled with care as it inserts superiorly to the paper-thin lateral edge of the cribriform plate. Excessive manipulation of this turbinate can fracture the cribriform plate and cause a cerebrospinal fluid leak.

- During frontotemporal intracranial approaches to the orbit, coagulation, and incision of the meningoorbital band will allow dissection retraction of the temporal dura from the cavernous dura and therefore improved exposure of the superior orbital fissure and anterior cavernous sinus region.

- Understanding the course of the frontal branch of the facial nerve combined with surgical techniques, such as interfascial or subfascial temporal muscle dissection, subperiosteal dissection on the zygomatic arch, and to avoid excessive inferior reflection of the scalp flap, can protect the nerve from inadvertent injury and consequent postoperative frontalis muscle palsy after anterolateral approaches to the orbit.

the ethmoid bone, also called lamina papyracea, and the body of the sphenoid. The quadrangular orbital plate of the ethmoid separates the orbit from the nasal cavity and it articulates superiorly with the medial edge of the orbital plate of the frontal bone. The anterior and posterior ethmoidal canals are formed by the anterior and posterior ethmoidal notches that exist in both of these plates ( - Fig. 1). These canals transmit the anterior and posterior ethmoidal branches of the ophthalmic artery and the nasociliary nerve of the ophthalmic division of the trigeminal nerve and open into the anterior cranial fossa along the lateral edge of the cribriform plate. $^{4}$ The cranial openings of the ethmoidal canals are located at the anterior and posterior limits of the ethmoidal cribriform plate and represent two important surgical landmarks for endonasal approaches to the anterior fossa and orbit; they divide the anterior fossa floor and nasal cavity roof into frontal (anterior to the anterior ethmoidal canal), cribriform (between both canals), and planum areas (posterior to the posterior ethmoidal canals), and similarly, the orbit into bulbar, retrobulbar, and apical parts. ${ }^{1}$ The anterior ethmoidal artery runs along the cranial base after exiting the orbit from posterior to anterior toward the cribriform plate. The anterior and posterior ethmoidal arteries may be totally covered by bone or freely hanging into the ethmoidal labyrinth, placing them at risk of inadvertent injury during surgery. ${ }^{5}$ The posterior ethmoidal artery is usually smaller than the anterior and may be absent in $30 \%$ of the cases. The anterior ethmoidal canal may be found on average $21-\mathrm{mm}$ posterior to the medial orbital rim. The posterior ethmoidal canal is located approximately $14-\mathrm{mm}$ posterior to the anterior ethmoidal canal, and the optic canal can be found on average $7-\mathrm{mm}$ posterior to the posterior ethmoidal canal (- Fig. 1 , part 1$)^{1}$

Besides contributing to the medial orbital wall, the cubical-shaped ethmoid bone also contributes to the nasal septum (perpendicular plate) and nasal cavity roof (cribriform plate).

On the lateral nasal wall, the supreme (if present), superior, and middle turbinates form part of the ethmoid bone, while the inferior turbinate is a separate bone, the inferior concha. The turbinates' major axes follow an oblique direction, with the posterior part lower than the anterior part. ${ }^{6,7}$ The middle turbinate insertion constitutes an important surgical landmark and follows three different planes: superiorly, the anterior third attaches to the lateral end of the cribriform plate in a sagittal plane; the middle third turns laterally to lie in a coronal plane (basal lamella) and attaches to the lamina papyracea; and the posterior third is attached to palatine bone, after rotating inferiorly to become horizontal. ${ }^{8}$ Therefore, the middle turbinate must be handled with great care as is attached to the paper-thin cribriform plate above and excessive manipulation can cause a fracture and consequent cerebrospinal fluid leak. Up to $35 \%$ of the patients may present a pneumatized middle turbinate. This anatomical variant, known as concha bullosa, is important from a surgical perspective as it may decrease the surgical working area. ${ }^{9}$

Beneath each of the nasal turbinates, the corresponding meatuses can be identified.

Two important ethmoidal structures are located in the middle nasal meatus, a bulging elongated osseous structure anteriorly called the uncinate process and apneumatized structure posteriorly that corresponds to the ethmoidal bulla. $^{8}$

The uncinate process is a thin hook-shaped bone projection from the ethmoid labyrinth.

Superiorly, the uncinate process may attach to the lamina papyracea, anterior skull base, middle nasal turbinate, or a combination of these. Anteriorly, the uncinate process attaches to the posterior edge of the lacrimal bone and inferiorly, to the ethmoidal process of the inferior nasal concha. Its posterior edge is free and is covered by mucosa. ${ }^{6}$

Posterior and above the uncinate process is the ethmoidal bulla, the largest and most constant of the ethmoid cells. The bulla is formed by the pneumatization of the bulla lamella or second ethmoid basal lamella (-Fig. 1, part 1). The retrobullar recess is a space that may be present between the posterior surface of the bulla ethmoidalis and the basal lamella of the middle turbinate, and the suprabullar recess may be present if the bulla does not reach the skull base. ${ }^{8}$

Between the anterior face of the bulla and the posterior edge of the uncinate process there is a two-dimensional crescent-shaped opening called the inferior semilunar hiatus. This semicircular space continues laterally as a three- 

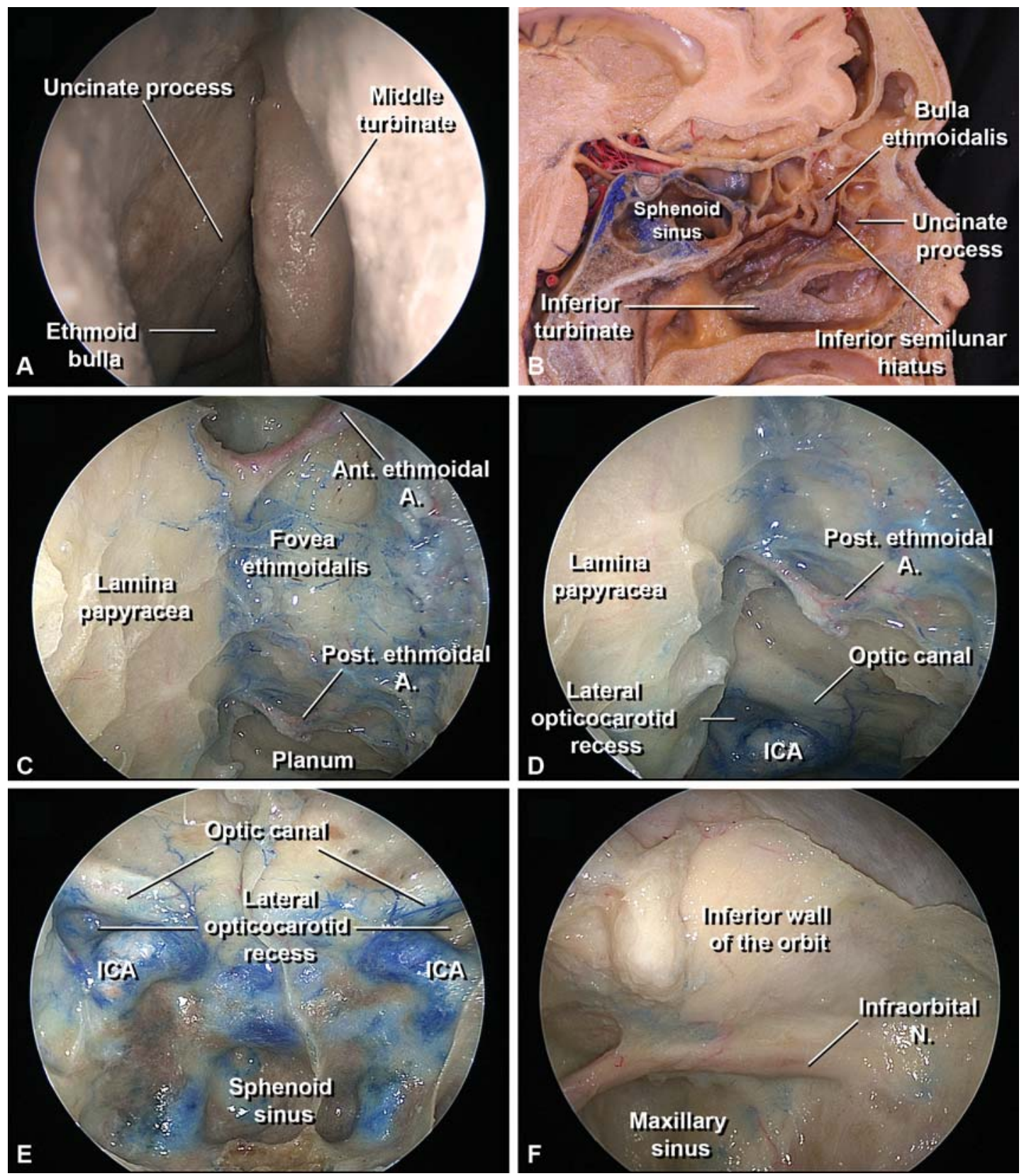

Fig. 1 Medial and inferior anatomical relationships of the orbit. The orbit from an endoscopic endonasal perspective, 0-degree endoscopic view.

(A) Identification of the right ethmoid bulla and uncinate process in the middle nasal meatus after careful medial displacement of the middle nasal turbinate. (B) Sagittal dissection of the left nasal cavity, the middle turbinate has been completely removed. The bulla ethmoidalis and the uncinate process have been partially divided. The space between the uncinate process and the bulla is the inferior semilunar hiatus. (C) The anterior and posterior ethmoidal canals with the respective arteries are visualized at the superior edge of the lamina papyracea and cranial base after removal of the ethmoidal cells. These canals divide the anterior fossa floor and nasal cavity roof into frontal (anterior to the anterior ethmoidal canal), cribriform (between both canals), and planum areas (posterior to the posterior ethmoidal canals), which approximately correspond to the to the bulbar, retrobulbar, and apical parts of the orbit. (D) Relationship of the posterior ethmoidal canal with the orbital apex posteriorly: the optic canal prominence is located superior to the lateral opticocarotid recess, a triangle-shaped depression superolateral to the carotid prominence. The lateral opticocarotid recess corresponds to the optic strut base intracranially, and the clinoid segment of the internal carotid artery is located medially. (E) Endonasal view of the posterior wall of the sphenoid sinus. (F) The inferior wall of the orbit corresponds to the superior wall of the maxillary sinus. The infraorbital nerve crosses through the infraorbital canal until it exits via the infraorbital foramen located in the inferior orbital rim. (G) Close view of the lamina papyracea. The medial wall of the maxillary sinus has been removed but the underlying mucosa has been preserved. (H) The lamina papyracea has been resected exposing the periorbita and the mucosa of the medial wall of the maxillary sinus has been removed. (I) The periorbita underneath the lamina papyracea has been incised and the orbit fat has been removed, exposing the neurovascular orbital contents through the medial orbit wall. (J) The superior wall of the maxillary sinus has been removed medially to the infraorbital nerve and the periorbita has been incised, exposing the neurovascular orbital contents through the medial and inferior orbit walls. Note how the infraorbital branch of the internal maxillary artery follows the infraorbital nerve at the infraorbital groove and receives an anastomosis from the ophthalmic artery. (K): Detailed view of the orbital apex and the optic canal. (L) Specimen divided in the sagittal plane, the ethmoid labyrinth and the medial wall of the maxillary sinus have been removed exposing the infraorbital canal and the orbital plate of the ethmoidal bone (lamina papyracea). A., Anterior; ICA, internal carotid artery; M, muscle; N, nerve; Post, posterior. 

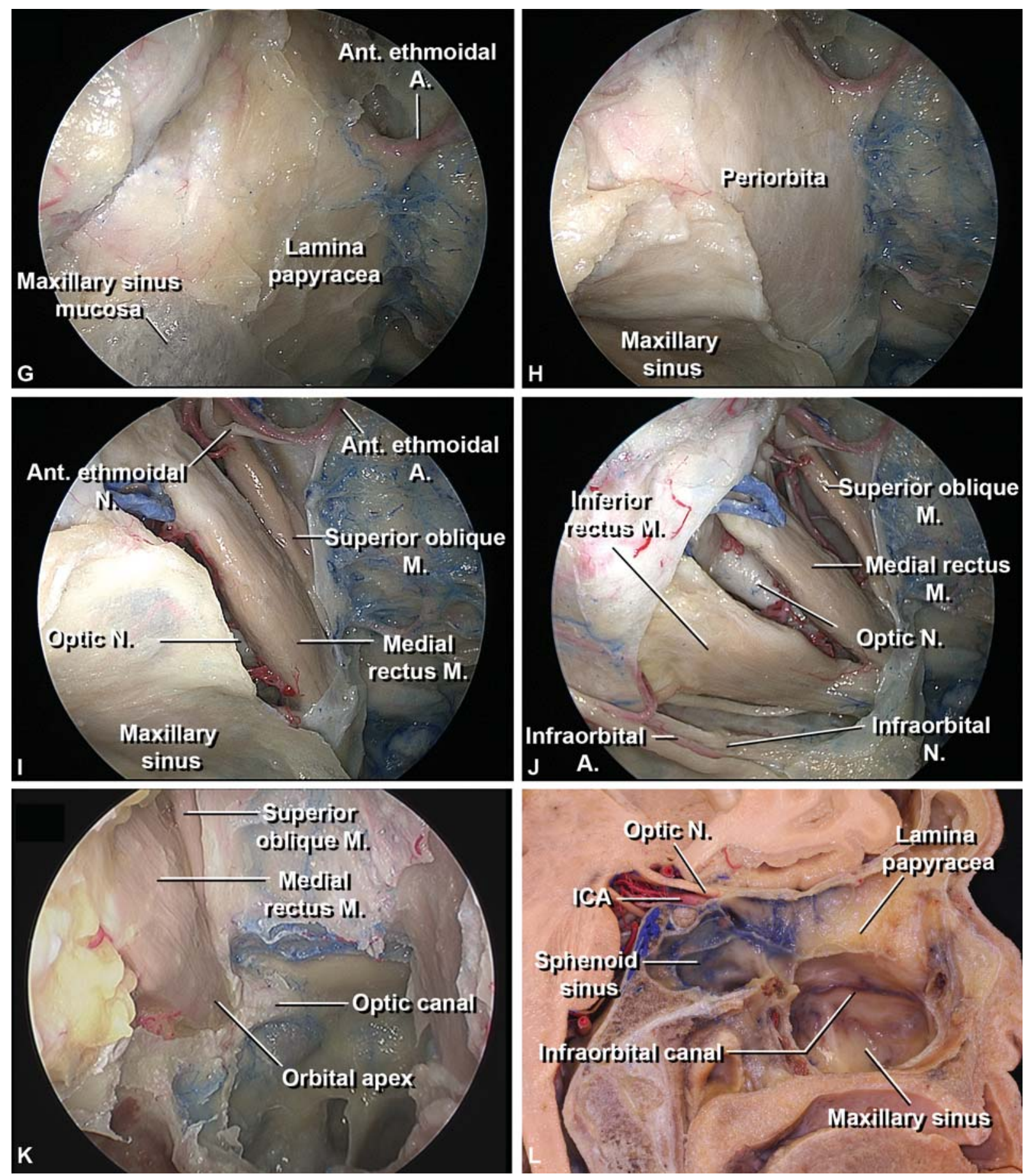

Fig. 1 (Continued)

dimensional slit-like space called the ethmoidal infundibulum. ${ }^{6}$ The ethmoidal infundibulum is bordered medially by the uncinate process and inferior semilunar hiatus, laterally by the lamina papyracea, inferiorly by the maxillary sinus ostium, and posteriorly by the ethmoidal bulla. The anterior ethmoidal cells also drain into the ethmoidal infundibulum, and in $50 \%$ of cases, the frontal sinus. On the other hand, the posterior ethmoidal cells drain into the superior meatus. ${ }^{7}$ The basal lamella of the middle turbinate is an important surgical landmark as it serves as a clear separation between the anterior and posterior ethmoid air cells. ${ }^{8}$
Once the uncinate process is excised, the ethmoidal bulla and the basal lamella of middle turbinate constitute the main surgical corridor to the posterior ethmoid and eventually to the orbital plate laterally.

The ethmoidal labyrinths consist of numerous paper-thin ethmoidal air cells. The medial surface of the labyrinth forms part of the superolateral nasal wall from the inferior surface of the cribriform plate to the middle turbinate. The lateral surface of the labyrinth forms part of the medial orbital wall (lamina papyracea; - Fig. 1, part 2). ${ }^{6}$ The roof of the ethmoidal labyrinth is called the fovea ethmoidalis and joins the 
cribriform plate through a thin piece of bone called the lateral lamella. ${ }^{8}$ The relationship of the fovea ethmoidalis with the lateral lamella and cribriform plate in the coronal plane can present many variations. $^{7}$

The posterior ethmoidal air cells are often situated immediately in front of the optic canal which is located on an average of 7-mm posterior to the posterior ethmoidal canal. ${ }^{1}$ Removing the posterior ethmoid sinus and the adjacent part of the sphenoid sinus will expose the medial wall of the optic canal (-Fig. 1). ${ }^{10}$ When the most posterior ethmoid cell pneumatizes superiorly and posteriorly above the sphenoid sinus, this posterior ethmoid cell is called a sphenoethmoidal cell or Onodi's cell. This is important from a surgical perspective as the optic nerve may be intimately related to its lateral wall and it places the optic nerve at increased risk of injury during posterior ethmoidectomy. ${ }^{8}$

\section{Inferior Relationships: The Maxillary Sinus and Related Structures}

The maxillary sinus is the entrance to several important surgical corridors, and its medial and superior wall provide access to the inferomedial wall of the orbit. The transmaxillary approach was traditionally performed using a sublabial incision in the gingivobuccal sulcus (Caldwell-Luc approach). ${ }^{11}$ Over the last several decades the endoscopic endonasal route has been added to the surgical armamentarium, either replacing previous transmaxillary approaches or utilizing endoscopic assistance after a Caldwell-Luc approach. ${ }^{12}$

The pyramid-shaped maxillary sinus, the largest of the paranasal sinus, is located in the body of maxilla. The floor is formed by the alveolar and palatine processes of the maxilla.

The anterior wall is formed by the facial surface of the maxilla and contains the canalis sinuosus, a groove for the anterior superior alveolar nerve and vessels. The roof of the maxillary sinus forms the inferior wall of the orbit and is traversed by the infraorbital nerve in its groove and canal. ${ }^{8}$ The posterior wall is formed by the infratemporal surface of the maxilla and forms the anterior border of the pterygopalatine fossa. This posterior wall contains the alveolar canals which transmit the posterosuperior alveolar nerves to the molar teeth. The lateral apex extends into the zygomatic process of the maxilla. ${ }^{13}$ The medial wall separates the sinus from the nasal cavity and is the site of the maxillary hiatus, the natural sinus opening that will provide surgical entrance to access the orbit through this corridor. The maxillary hiatus is located just below the roof of the sinus and opens into the inferior part of the of the ethmoidal infundibulum, between the middle and posterior thirds of it, at the middle meatus. ${ }^{8}$ This opening is partially closed by the uncinate process of the ethmoid bone, its margins should be identified and removed as a first step in performing the antrostomy to widen the access to the maxillary sinus (-Fig. 1 ). The root of the middle nasal concha attaches to the lateral nasal wall near the junction of the orbit and the maxillary sinus. ${ }^{7,8}$

The infraorbital nerve arises from the maxillary division of the trigeminal nerve at the pterygopalatine fossa. After entering the orbit via the inferior orbital fissure (IOF), it usually courses anteriorly through the infraorbital groove and canal. The infraorbital canal is located anteriorly within the orbital floor or superior wall of the maxilla. The infraorbital nerve then exits the canal through the infraorbital foramen of the anterior maxilla (-Fig. 1) ${ }^{6,10}$ Sometimes, however, the infraorbital canal protrudes into the maxillary sinus separate from the orbital floor. This variant may lead to inadvertent nerve injury during endoscopic surgery. The infraorbital nerve traversing the lumen of the maxillary sinus can be found in more than $10 \%$ of the patients (-Fig. 1F) and is even more prevalent in the setting of another anatomical variant, an ipsilateral infraorbital ethmoid cell (Haller's cell), especially if the nerve is contained within the lamellae of the cell. ${ }^{14}$ This ethmoidal cells that develop into the maxillary sinus may narrow the drainage pathway of the maxillary ostium or the infundibulum ethmoidal and also obstruct distal regions, narrowing the space for the passage of the endoscope and surgical instruments. ${ }^{8}$ Preoperative image studies can help to identify these variants and avoid nerve damage.

During certain surgical procedures, inadvertent damage to the nasolacrimal apparatus may occur as well. ${ }^{15}$ The lacrimal drainage pathway includes the superior and inferior lacrimal canaliculi, the lacrimal sac, and the nasolacrimal duct. The lacrimal sac lies in the nasolacrimal groove at the inferomedial aspect of the orbit, beneath the medial canthal ligament. Endonasally this region corresponds to the area just anterior to the origin of the axilla of the middle turbinate. ${ }^{10}$ The nasolacrimal groove is formed anteriorly by the frontal process of the maxilla and posteriorly by the lacrimal bone. ${ }^{13}$ The medial canthal ligament is firmly attached to the anterior and posterior margins of the lacrimal groove. If a medial orbital approach is performed, the medial canthal ligament should be sharply divided or elevated in such a way that it can be preserved for appropriate reattachment during the closure. ${ }^{10}$ The lacrimal sac drains inferiorly through the nasolacrimal duct which opens into the inferior meatus, approximately $2 \mathrm{~cm}$ behind the nostril. ${ }^{7}$ Care must be taken during the uncinectomy and maxillary antrostomy to avoid injury to the nasolacrimal duct. ${ }^{15}$

\section{The Orbit from a Posterior and Superolateral Intracranial Perspective}

\section{Superior Relationships: The Orbitofrontal Cortex and Surrounding Dura Overview}

The orbitofrontal cortex, also known as ventromedial prefrontal cortex, lies immediately above the orbits (-Fig. 2). Although considerable individual variability has been found, the orbitofrontal cortex is usually formed by the lateral, medial, anterior, and posterior orbital gyri, and the orbital sulci.

The medial and lateral orbitofrontal arteries provide the blood supply to the orbitofrontal cortex. The medial orbitofrontal artery is the first branch of the A2 segment of the anterior cerebral artery. It branches distal to the anterior communicating artery and passes anteriorly and inferiorly toward the frontobasal surface of the olfactory sulcus to reach the level of the planum sphenoidale. It terminates at 

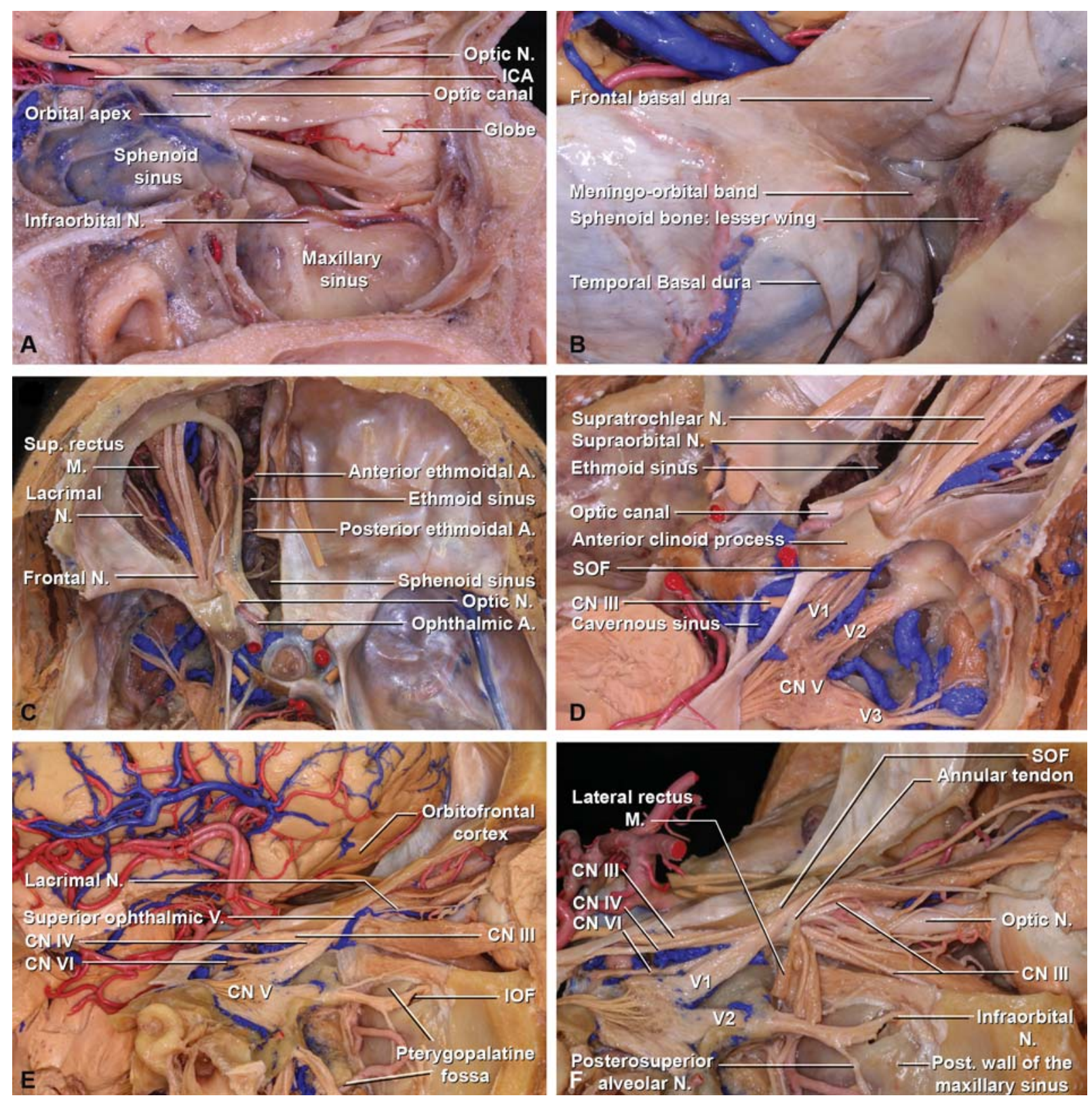

Fig. 2 Lateral and superior anatomical relationships of the orbit. (A) Sagittal view of the medial left orbit. The orbital plate of the ethmoidal bone (lamina papyracea), the medial wall of the maxillary sinus and the superior wall of the maxillary sinus medially to the infraorbital nerve have been removed. The periorbita have been excised and the orbital fat has been resected. The intracranial portion of the optic nerve and the ophthalmic artery are observed entering the optic canal. (B) A frontotemporal craniotomy has been performed on the right side and the lesser wing of the sphenoid bone has been drilled. The meningoorbital band, a dural fold located on the lateral side of the SOF, is exposed. Note how this dura bridge tethers the frontotemporal basal dura to the periorbita, and therefore should be carefully incised to allow further exposure of the SOF, paraclinoid, and cavernous sinus region. (C) View of the orbit and cranial base from superior, the brain has been resected. On the left side, the dura covering the anterior and middle fossa floor has being excised, the superior wall of the frontal and ethmoid sinuses, the orbital roof, superior periorbita, and orbital fat have being resected. (D) Right oblique intracranial view. The dura, brain, superior wall of the ethmoid sinus, superior and lateral orbit walls, periorbita, and orbit fat have being excised. The structures located in the SOF, IOF and the main contents of the cavernous sinus are demonstrated. Note how the SOF superior is posteriorly related to the cavernous sinus and the optic canal opens medially into the intradural space. (E) Right lateral intracranial view. The neurovascular structures accessing the orbit through the SOF and IOF are seen. The orbitofrontal cortex, also known as ventromedial prefrontal cortex, lies immediately above the orbits. The lateral compartment of the SOF constitutes the corridor through which the superior ophthalmic vein, the trochlear, frontal and lacrimal nerves enter the orbit. The central compartment, situated just behind the annular tendon, transmits through this tendinous ring the superior, and inferior divisions of the oculomotor nerve, the abducens and nasociliary nerves, and the sensory and sympathetic roots of the ciliary ganglion. The IOF opens into the temporal, infratemporal, and pterygopalatine fossae. (F) Right lateral intracranial view. The lateral rectus muscle has been divided and retracted laterally. The infraorbital nerve arises from the maxillary division of the trigeminal nerve at the pterygopalatine fossa and enters the IOF. A, artery; CN, cranial nerve; ICA, internal carotid artery; IOF, inferior orbital fissure; M, muscle; N, nerve; SOF superior orbital fissure, $V$ vein. 
the orbitofrontal cortex, supplying the orbital gyri and inferomedial portion of the frontal lobe. The lateral orbitofrontal artery most frequently branches off the superior trunk of the middle cerebral artery at the M2 segment. It passes between the frontal and temporal lobes from posteroinferior to anterosuperior to arrive at the posterolateral orbitofrontal cortex surface, where it primarily supplies the lateral orbitofrontal cortex. A remarkable variability in the number of their branches and anastomoses has been described. $^{6}$

When either a frontotemporal or a supraorbital craniotomy are the selected routes to approach the orbit, the frontal dura that covers the orbitofrontal cortex and the related orbitofrontal arteries has to be carefully dissected free from the orbital roof and the cortex gently retracted. The orbitofrontal cortex is connected with sensory regions, as well as limbic system structures, playing an important role in memory, emotion, and the cognitive process of decision making; inadvertent surgical damage, although uncommon during orbital surgery, may lead to a pattern of disinhibited behavior. $^{16}$

The dural layers and their organization around this area are complex and relevant in surgery. The intracranial dura is a thick collagenous sheath composed of a periosteal layer that faces the bone and a meningeal layer that faces the brain. These layers are distinguished as separate sheaths at the venous sinuses and optic canal. The meningeal layer fuses with the epineurium of the cranial nerves and with the adventitia of the vessel as they emerge from the cranium through the cranial foramina or fissures. An exception is the optic nerve in which, after conforming the optic nerve sheath, the meningeal layer blends into the outer scleral layers. The periosteal layer of dura is continuous with the pericranium through the cranial sutures and foramina and with the periorbita through the superior orbital fissure (SOF) and optic canal. ${ }^{6}$ Hence, at the SOF, the dura covering the middle fossa and cavernous sinus (CS) blends into the periorbita of the orbital apex, but also into the annular tendon from which the rectus muscles arise. ${ }^{17}$ The annular tendon, also called the annulus of Zinn, adheres to the dural sheath of the optic nerve and it is attached to the periosteum along the upper, medial, and lower margins of the optic canal; its lower portion extends horizontally from the sphenoid body below the optic foramen and optic strut to attach to a bony prominence at the midportion of the lateral edge of the SOF. ${ }^{13}$ Consequently, when the frontotemporal basal dura is fully retracted, a dural fold is formed on the lateral side of the SOF. This superficial dural bridge that tethers the frontotemporal basal dura to the periorbita, named by some authors the meningoorbital band, contains few a small dural veins and the orbitomeningeal artery (-Fig. 2 ). ${ }^{18}$ Coagulation and incision of this dura fold will allow sufficient retraction of the temporal dura and therefore improved exposure of the SOF, which is potentially the most posterior limit of the superior orbitotomy, and will also provide a wider exposure for approaching the paraclinoid and anterior CS region. Once the dura fold is carefully incised and detached from the periorbita, the dura of the temporal lobe can then be dissected from the middle fossa floor to proceed medially peeling the outer layer (periosteal dura) away from the inner layer (meningeal layer) of the $\mathrm{CS}$, exposing the cranial nerves that pass through the CS on their course to the orbit when the surgery requires it. ${ }^{18,19}$

The meningoorbital foramen, also called cranioorbital foramen, is located in the greater wing of the sphenoid, usually anterior to the lateral aspect of the SOF, but it can be lateral or even confluent with its lateral end, and it can have multiple openings in up to $15 \%$ of the cases. ${ }^{20}$ The cranioorbital foramen connects the middle cranial fossa with the orbit and contains an anastomosis between the orbital branch of the middle meningeal artery, known as meningolacrimal artery, and a recurrent meningeal branch of the lacrimal artery that exits the orbit through the SOF, courses laterally below the sphenoid ridge, and turns forward through the cranioorbital foramen to supply the periorbita. ${ }^{10}$ This foramen is present in approximately $55 \%$ of the cases, while in the remaining cases the anastomotic ramus usually enters the orbit through the SOF. ${ }^{20}$ This small foramen is important from a surgical perspective because it constitutes a potential source of hemorrhage that surgeons must take into account during deep orbital dissection and its intraoperative identification could avoid unexpected bleeding that may complicate surgery at the orbital apex. $^{21}$

\section{Posterior and Posteroinferior Relationships}

The three important osseous openings that connect the orbit with the intracranial and extracranial spaces are the SOF and IOF and the optic canal. The SOF is posteriorly related to the $\mathrm{CS}$, the optic canal opens posteromedially into the intradural space and the IOF opens into the temporal, infratemporal, and pterygopalatine fossae.

\section{Superior Orbital Fissure and Cavernous Sinus}

The SOF is a bony cleft located between the orbital apex anteriorly and the CS posteriorly, constituting a potential route of extension of orbital lesions toward the CS and middle cranial fossa and vice versa. Its lateral aspect is narrow and is formed by the lesser and greater wing of the sphenoid bone and a small portion of the frontal bone at its lateral apical margin, whereas its medial edge is wider and is formed by the sphenoid body. The lower end of the SOF blends inferiorly with the posterior end of the IOF. The superior margin of the SOF is delimited by the lower surfaces of the lesser wing, the optic strut, and the anterior clinoid process. The lower margin, delimited by the junction of the greater wing with the sphenoid body, is located at the level of the lower edge of the CS, with some of its venous spaces extending forward along these margins. The lower margin is separated from foramen rotundum by a bony bridge, the maxillary strut (-Figs. 2, 3). ${ }^{10,17}$

The annular tendon is located in the anterior upper half of the medial part of the fissure and divides the fissure into three compartments: lateral, central, and inferior. The lateral compartment is situated at the lateral aspect of the SOF outside the annular tendon and constitutes the corridor through which the superior ophthalmic vein, the trochlear, frontal, and lacrimal nerves enter the orbit. The central compartment, situated 

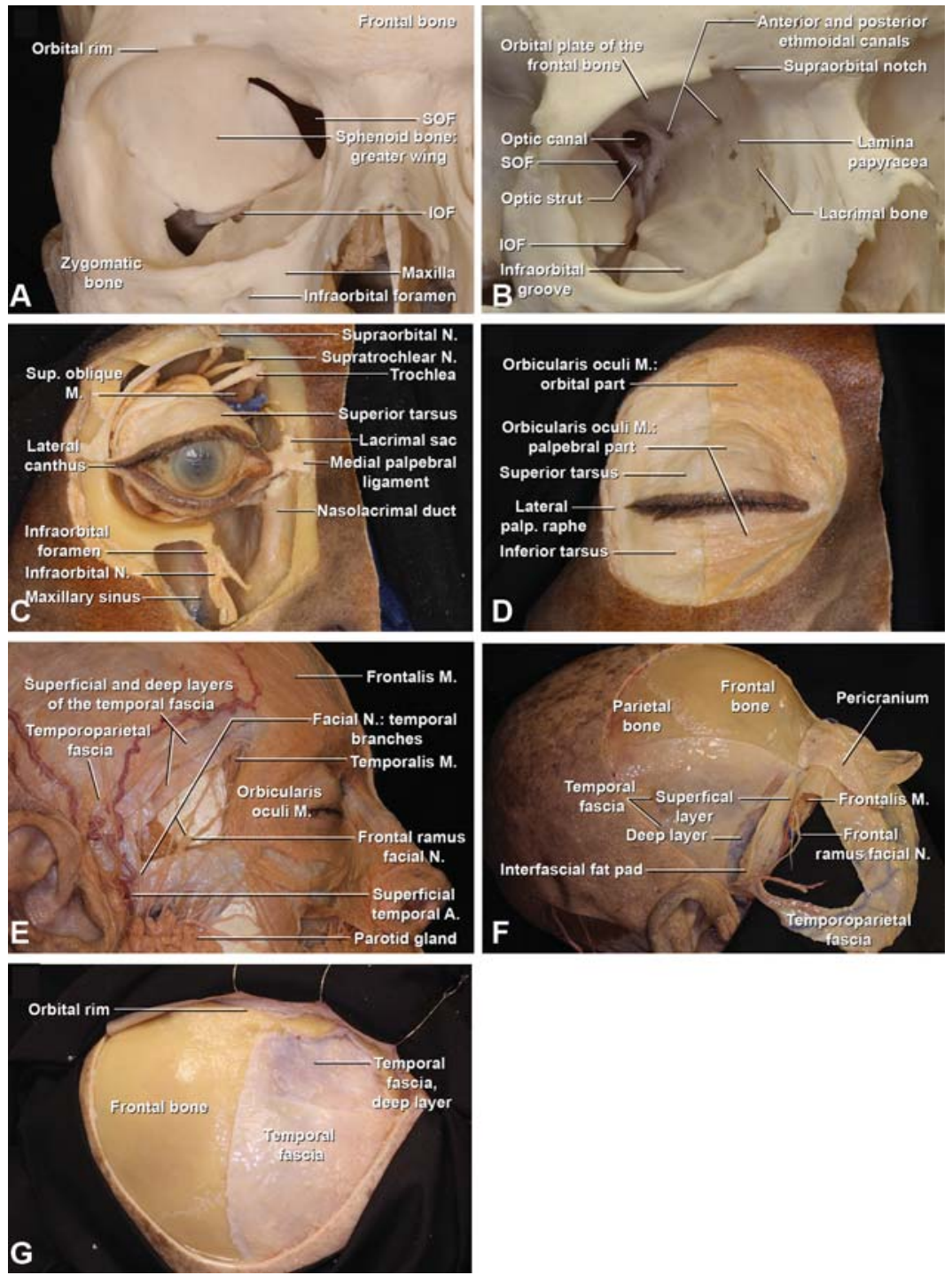

Fig. 3 Anterior anatomical relationships of the orbit. (A, B) A anterior and oblique overview of the osseous components of the right orbit, which is formed by seven different bones: the frontal, zygomatic, sphenoid, lacrimal, ethmoid, palatine bones, and maxilla. Note the paper-thin consistency of the lamina papyracea, and its relationship with the anterior and posterior ethmoidal canals, within the superior edge of the lamina papyracea and the medial edge of the orbital plate of the frontal bone. The canals offer exit to the anterior and posterior ethmoidal arteries (which are branches of the ophthalmic artery) and the nasociliary nerve (division of the ophthalmic nerve). The nasolacrimal groove is formed anteriorly by the frontal process of the maxilla and posteriorly by the lacrimal bone. The optic canal is observed close to the posterior ethmoidal canal and contains the optic nerve, ophthalmic artery and sympathetic nerve fibers. The SOF and IOF are identified. Note how the lower end of the SOF blends inferiorly with the posterior end of the IOF. Note the infraorbital foramen of the anterior maxilla and the supraorbital notch at the supraorbital rim. (C) Oblique view of the right orbit. The skin surrounding the orbit, extraocular muscles and orbital fat have been removed, and the maxillary sinus opened. The medial and lateral canthus are demonstrated. The supra-orbital nerve emerges from the supra-orbital foramen and the supratrochlear nerve is located medially between the supraorbital foramen and the trochlea of the superior oblique muscle. The inferior orbital wall has been removed medially up to the infraorbital canal. The infraorbital nerve leaves the orbit through the infraorbital foramen and divides into the palpebral, nasal and superior labial branches, supplying sensory innervation of the skin of the lower eyelid, medial cheek, lateral nose and upper lip, and the mucosa of the anteroinferior nasal septum and oral mucosa of upper lip. (D) Right anterior view of the orbit after skin removal. The orbital and palpebral portions of the orbicularis oculi muscle are observed in the medial half of the orbit. The lacrimal part of the orbicularis oculi muscle lies underneath these structures, extending behind the lacrimal sac and attaching to the lacrimal bone. The orbicularis oculi muscle surrounds the orbital rim circumferentially and extends into the lids, temple and cheek, having its fibers interdigitated with the occipitofrontalis and the corrugator muscles. On the lateral side of the orbit, the orbicularis oculi muscle has been resected and the two dense plates of connective tissue know as tarsi are observed. (E) Right lateral view to illustrate the course and location of the temporal branches of the facial nerve. The temporal division of the facial nerve emerges directly through the parotid gland and divides in three rami: anterior, middle and posterior. The temporal branches are located superficial to the temporoparietal fascia (also known as superficial temporal fascia). The middle ramus (frontal ramus), which innervates the frontalis muscle, is located anteroinferior to the frontal branch of the superficial temporal artery. The frontalis muscle is continuous laterally with the temporoparietal fascia and is medial to the superior temporal line. (F) Dissection of the right temporal area. The temporoparietal fascia and the frontalis muscle have been reflected anteriorly and part of the temporoparietal fascia has been resected to expose the frontal ramus of the facial nerve and the superficial temporal artery that run on this layer. The temporal fascia is continuous with the pericranium medially and divides 2 to $3 \mathrm{~cm}$ above the zygomatic arch into two layers, superficial and deep. In between these layers the interfascial fat pad is encountered. To protect the frontal ramus of the facial nerve the dissection of the skin flap is performed between the deep and superficial layers of the deep temporal fascia (interfascial dissection) or under the deep layer (subfascial dissection). (G): Right frontotemporal exposure within trifacial temporal dissection. The interfascial-subpericranial flap has been folded anteriorly. A, artery; IOF, inferior orbital fissure; M, muscle; N, nerve; Palp, palpebral; SOF, superior orbital fissure. 
just behind the annular tendon, transmits through this tendinous ring the superior and inferior divisions of the oculomotor nerve, the abducens and nasociliary nerves, and the sensory and sympathetic roots of the ciliary ganglion. The inferior compartment is located below the annular tendon and transmits the inferior ophthalmic vein. This compartment contains some of the orbital fat that extends backward into the fissure and in its lower margin a posterior extension of the orbital smooth muscle. ${ }^{10,17}$ The orbital smooth muscle constitutes a landmark to reach the SOF from an endoscopic endonasal perspective, therefore also useful for CS and orbital apex regions approaches. ${ }^{22}$

When accessing the lateral segment of the SOF, the opening should be attempted through its upper margin, as the superior ophthalmic vein and the large Sylvian veins that empty into the CS are located at the lower margin of it. Special care must be taken to not damage the trochlear nerve, which passes along its upper margin above the ophthalmic nerve. $^{17}$

The posterior SOF transmits and is related to the CS. The CS is a vascular structure defined traditionally as a fourwalled dural envelope that runs from the SOF and anterior clinoid process (ACP) anteriorly to above the petrous apex and posterior clinoid process posteriorly. ${ }^{23}$ It lies lateral to the dorsum sellae and sphenoid body and medial to the junction of the greater wing of the sphenoid bone. This venous plexus maintains connections to the superior and inferior ophthalmic veins, superior and inferior petrosal sinuses, basilar sinus, and middle and inferior cerebral veins.

The main arterial structure encased in the CS is the intracavernous portion of the internal carotid artery (ICA), which is accompanied and surrounded by a sympathetic nerve plexus. The ICA enters the CS after passing under the petrolingual ligament, follows an ascending course and forms a posterior bend lateral to the posterior clinoid process. The ICA then follows a horizontal trajectory and forms a bend anteriorly. The ICA leaves the CS through its superior wall medial to the anterior clinoid process. The intracavernous ICA gives rise to branches that supply the structures contained in the CS, sellae, and tentorium. These branches display some degree of variability, with the most consistent being the meningohypophyseal and inferolateral trunk. The meningohypophyseal trunk, the largest intracavernous branch, usually arises from the posterior bend of the intracavernous ICA and further divides into three branches: the tentorial, dorsal meningeal and inferior hypophyseal arteries. The inferolateral trunk usually arises from the lateral portion of the ICA as it courses anteriorly. ${ }^{24}$

The III, IV, and VI cranial nerves and the first division of the $V$ nerve also pass through the CS on their course to the SOF. The VI cranial nerve is located completely inside the CS, medial to the ophthalmic division of the V nerve. The III, IV, and $\mathrm{V} 1$ nerves lie within the dura of the lateral sinus wall (-Fig. 2). ${ }^{25,26}$

\section{Optic Canal}

The optic canal is located in the sphenoid bone medial to the SOF extending in a superior medial direction from the orbital apex to the optic foramen (-Figs. 2 and $\mathbf{3}$ ). The optic canal is funnel-shaped, narrower anteriorly near the orbit, and is bounded laterally by the lesser wing of the sphenoid and anterior clinoid process and medially by the body of the sphenoid. ${ }^{27}$ The anterior root of the lesser wing of the sphenoid forms the roof of the optic canal and the posterior root or optic strut forms the floor. ${ }^{10}$ The optic strut, or posterior root of the lesser wing of the sphenoid bone, is a bony bridge running between the sphenoid bone and the lower medial margin of the base of the anterior clinoid process. ${ }^{25}$ It separates the SOF from the optic canal and foramen, conforming the junction of the upper and medial walls of the SOF and the lateral edge and floor of the optic canal. ${ }^{13}$ The inferior surface of the optic strut also forms the anterior part of the roof of the CS. ${ }^{19}$

The optic canal transmits the optic nerve, ophthalmic artery, and sympathetic nerve fibers. The optic nerve follows a superomedial trajectory from the orbit, whereas the ophthalmic artery is located inferomedially within the proximal portion of the canal and inferolaterally within the distal portion. ${ }^{1}$ The ophthalmic artery constitutes the major supply to the orbit and in the majority of the cases is a branch of the supraclinoid portion of the ICA. However, instead of always arising in the subarachnoid space, the ophthalmic artery can arise in the CS, be a branch of the middle meningeal artery or even arise as duplicate arteries of nearly equal size (-Fig. 2$)^{1,24}$

The walls of the canal can be thinned by the proximity of the sphenoid sinus, and sometimes if the sphenoid sinus is well pneumatized, the medial bony layer may be even absent, leaving the optic nerve separated from the sinus by only the dural nerve sheath and mucosa. ${ }^{28}$ This anatomic variation should be carefully addressed in preoperative images and kept in mind especially during endoscopic endonasal surgery, but also during intracranial approaches to prevent postoperative CSF leaks. If there is an Onodi's cell or sphenoethmoidal cell, the optic canal and often the ICA can be seen inside the cell and care must be taken not to injure them during an endonasal approach.

The portion of the optic nerve proximal to the optic foramen is covered by the falciform process or ligament, a reflected leaf of dura mater that extends medially from the anterior clinoid process across the top of the optic nerve to the sphenoid limbus. ${ }^{25}$ Compression of the optic nerve against the sharp edge of the falciform process due to an expanding lesion or surgical maneuvers may result in visual loss. The length of nerve covered just by the falciform process without underlying bone can vary from $1 \mathrm{~mm}$ to $1 \mathrm{~cm}$ and special care must be taken not to coagulate the falciform ligament and carefully divide it to release the optic nerve, as the lack of bone could lead to nerve injury. ${ }^{28}$ The trajectory of the ophthalmic artery in relation to the optic nerve at the optic canal must be also remembered to prevent iatrogenic lesion to this artery during opening of the falciform ligament. ${ }^{1}$

Once the optic nerves emerge from the optic foramina, they are directed posteriorly, superiorly, and medially toward the optic chiasm. The optic chiasm lies above and usually behind the chiasmatic sulcus, a groove connecting both optic foramina. ${ }^{28}$ The chiasmatic sulcus is located on 
the superior surface of the sphenoid bone, bounded anteriorly by the planum sphenoidale and posteriorly by the tuberculum sellae. ${ }^{10}$ From the chiasm, the optic tracts follow a posterolateral direction around the cerebral peduncles to enter the incisural spaces. ${ }^{28}$

\section{Inferior Orbital Fissure}

The IOF is located between the lateral wall and floor of the orbit posteriorly. The IOF has long anterior and posterior edges and narrow medial and lateral ends. The fissure is formed laterally by the zygomatic bone, posterolaterally by the greater wing of the sphenoid bone, anteriorly by the orbital surface of the maxilla, and a short segment of the orbital process of the palatine bone and medially by the sphenoid body. ${ }^{10}$

The anterolateral portion of the IOF communicates with the temporal fossa inferiorly. This portion contains only smooth muscle and adipose tissue, being a suitable point to perform the bony cuts needed to remove part of the roof, and lateral walls when performing frontotemporal or lateral approaches. ${ }^{1}$ The medial portion of the fissure connects the orbit to the infratemporal fossa that is located below the greater sphenoid wing. ${ }^{13}$ This segment contains the entrance of the infraorbital branch of the maxillary nerve and the infraorbital branch of the internal maxillary artery into the infraorbital groove. The posteromedial part of the IOF communicates below with the pterygopalatine fossa and, through this, with the nasal cavity ${ }^{1}$ (-Fig. 2).

Other neurovascular structures passing through the fissure are the zygomatic nerve and branches of the inferior division of ophthalmic vein that communicates with the pterygoid plexus. The zygomatic nerve branches off the maxillary nerve in the pterygopalatine fossa to enter the orbit by the IOF and to course along the lateral orbital wall, where it divides into zygomaticofacial and zygomaticotemporal branches, which exit the orbit through identically named foramina. ${ }^{10}$ The orbital smooth muscle (Müller's muscle) spans the upper entire length of the fissure and blends into the periorbita, perineurium of the infraorbital nerve, and periosteum of the maxillary bone, with some of its fibers reaching posteriorly the inferior wall of the CS and the SOF. ${ }^{22}$ The superior surface of the orbital smooth muscle is in intimate relationship with the inferior rectus muscle, inferior branch of the oculomotor nerve, and inferior ophthalmic vein. ${ }^{29}$

\section{The Orbit from an Anterior Perspective}

Direct access to the orbital contents with no need for bone removal can be achieved using either transcutaneous or transconjunctival approaches. While a pure transconjunctival approach gives access to the orbit through the conjunctiva of the inferior eyelid, the transcutaneous approaches can be used in the periorbital area including superior and inferior eyelids. The transconjuctival approach has the advantage of avoiding skin incisions; however, the exposure achieved may be limited. If an increased surgical corridor is required, a lateral canthotomy can be associated. The lateral canthotomy increases the risk of lower lid malpositionpostoperatively. ${ }^{30}$ In cases of transcutaneous approaches (superior palpebral/eyebrow incisions or inferi- or palpebral incisions: subciliary, subtarsal, or infraorbital), an understanding of the underlying muscular, neural, and tendinous anatomy is key.

The orbicularis oculi muscle lies just below the loosely attached layer of skin of the eyelids. The orbicularis oculi muscle surrounds the orbital rim and extends into the lids, temple, and cheek. There are three defined portions of the orbicularis muscle as follows: the orbital, palpebral, and lacrimal parts. The orbital portion lies circularly around the margins of the orbit and is the sphincter muscle of the eyelids. The palpebral portion is situated in the margins of the upper and lower eyelids and closes the eyelids. The lacrimal portion lies the most deep, extending behind the lacrimal sac, and attaching to the lacrimal bone, and is important in tear transport. ${ }^{6}$

The orbital portion arises from the medial palpebral ligament, nasal process of the frontal bone, and frontal process of the maxilla. Its lateral upper fibers blend with the occipitofrontalis and the corrugator muscles. Many of its upper fibers are inserted also into the skin and subcutaneous tissues of the eyebrow. The palpebral portion arises from the bifurcation of the medial palpebral ligament and inserts on the lateral palpebral raphe, which is a ligament located on the outer canthus of the eye (-Fig. 3 ). The lacrimal portion is located behind the medial palpebral ligament and arises from the posterior lacrimal crest and adjacent part of the orbital surface of the lacrimal bone. ${ }^{10}$

The tarsi, two dense plates of connective tissue that serve as the structural support of the eyelids, are located underneath the palpebral portion of the orbicularis oculi muscle.

The aponeurosis of the levatorpalpebræ muscle is attached to the anterior surface of the upper tarsus. The tarsi have rigid attachments to the periosteum through the canthal tendons medially and laterally. ${ }^{10}$ The medial ends of the tarsi are attached by the medial palpebral ligament to the frontal process of the maxilla in front of the lacrimal crest, crossing the lacrimal sac. As the ligament crosses the lacrimal sac, a strong aponeurotic lamina from its posterior surface expands over the sac to attach the upper part of the posterior lacrimal crest. ${ }^{31}$ The lateral ends of the tarsi are attached by the lateral palpebral ligament, a much weaker structure than the medial palpebral ligament, to a tubercle on the frontosphenoidal process of the zygomatic bone immediately within the orbital margin. ${ }^{21}$

The orbital septum is an extension of the periorbita that runs into the eyelid, separating the orbital contents from thelid contents. This membranous sheet extends to the tarsus from the orbital rim, where it attaches to the bone and becomes the periorbita inside the orbit and periosteum along the anterior edge of the orbit. ${ }^{21}$ In the upper eyelid, the septum blends with the aponeurosis of the superior levator muscle, and in the lower eyelid with the anterior surface of the tarsus. ${ }^{10}$

The medial canthus therefore consists of the attachment of the orbicularis oculi muscle and tarsus through the medial palpebral ligament, lacrimal sac, and a fibrous extension from the fascia, covering the medial rectus muscle attached to the lacrimal bone immediately posterior to the posterior lacrimal crest, known as medial check ligament. ${ }^{21}$ The lateral canthus consists of the attachment of the tarsus, the 
aponeurosis of the superior levator muscle and the lateral check ligament (a fibrous extension from the fascia covering the lateral rectus muscle) to the tubercle located on the zygomatic bone, whereas the fusion of the upper and lower orbicularis oculi occurs superficially at the lateral palpebral raphe (-Fig. $\mathbf{3}) .^{31}$

Eventually, when accessing orbital lesions requires an anterolateral craniotomy with supraorbital rim removal or lateral orbitotomy, knowledge and preservation of the temporal branches of the facial nerve and frontal branches of the trigeminal nerve is essential.

The frontal nerve arises from the ophthalmic division of the trigeminal nerve at the lateral wall of the $\mathrm{CS}$, passes through the lateral part of the SOF outside the annular tendon and courses over the levator muscle to divide into the supratrochlear and supraorbital nerves within the orbit. The supraorbital and supratrochlear nerves exit the orbit anteriorly through a notch or foramina in the supraorbital rim with the supraorbital and supratrochlear artery respectively, both branches of the ophthalmic artery. The supratrochlear nerve passes between the supraorbital foramen and the trochlea of the superior oblique muscle in a superior direction over the forehead (-Figs. 2 and 3). ${ }^{6,10}$ The supraorbital nerve may be released if necessary by removing bone from the lower margin of its foramen. Inadvertent injury of the supraorbital or supratrochlear nerve causes sensory disturbances of the forehead and scalp. The supraorbital notch constitutes an important surgical landmark for craniotomies that include removal of the supraorbital rim, like the orbitozygomatic and supraorbital approaches. The supraorbital notch has been traditionally used as medial craniotomy limit to avoid entry into the frontal sinus and supratrochlear nerve injury. ${ }^{32}$ However, its relationship with the frontal sinus and landmark reliability have been questioned. ${ }^{33}$ Careful study of a preoperative CT is mandatory to define the limits of the frontal sinus.

The temporal branch of the facial nerve arises inside the parotid gland, pierces the parotidomasseteric fascia below the zygomatic arch and then above the zygomatic arch divides into its three terminal rami: anterior, middle, and posterior. The middle ramus, also called frontal ramus, innervates the frontalis muscle. The frontalis muscle is medial to the superior temporal line, and is embedded within the galea (-Fig. 3). This layer is continuous laterally with the temporoparietal fascia (also known as superficial temporal fascia) over the area of the temporalis muscle. Deep to the temporoparietal fascia lies the temporal fascia (also known by some authors as deep temporal fascia), which divides into a deep and superficial layer 2 to $3 \mathrm{~cm}$ over the zygomatic arch. In between the superficial and deep layers of the deep temporal fascia lies the interfascial fat pad (also known as superficial fat pad). The frontal ramus of the facial nerve usually runs in the plane of the temporoparietal fascia; however, sometimes, the frontal ramus goes into the interfascial fat pad and then becomes again superficial to enter the frontalis muscle. ${ }^{34,35}$ When the skin flap is reflected anteriorly, and the temporalis muscle is reflected inferiorly to perform an anterolateral craniotomy, the temporalis muscle dissection can put these nerves in jeopardy. Although the majority of the cases have a good aesthetic result, injury of the frontal ramus may carry undesirable cosmetic defects as permanent or transient frontalis muscle palsy. To avoid frontal ramus damage, special care must be taken and different techniques like interfascial dissection (between the superficial and deep layers of the deep temporal fascia) or subfascial dissection (below the deep layer) have been proposed (-Fig. 3). ${ }^{32}$ When performing a supraorbital approach, injury to the frontal ramus should be also prevented. Dissection deep to the frontalis muscle close to the periosteum may prevent nerve damage. Other technical considerations, like subperiosteal dissection on the zygomatic arch, and avoidance of excessive inferior reflection of the scalp flap can also protect the frontal ramus from inadvertent injury. ${ }^{36}$

\section{Reconstructive Options}

Usually endoscopic endonasal approaches to the orbit with removal of its medial wall do not require reconstruction. At the end of the procedure, an absorbable packing can be placed in the ethmoid area and would prevent even rare enophthalmos develop after surgery. On the other hand, if the orbital floor is widely removed and the fat is herniating into the maxillary sinus, reconstruction of the orbital floor is recommended due to high risk of enophthalmos. Synthetic implants can be placed endonasally or an external approach may be required for the reconstruction. ${ }^{37}$ Transient diplopia after orbital surgery is very frequent and usually resolves within a month if there is no neural or muscular damage. If there is an adjacent dural defect (in the ethmoidal or frontal area, for example), the repair can be performed with free mucosal graft, nasoseptal flap or pericranial flap depending on the size and location of the intracranial defect. In cases of orbital exenteration, temporalis muscle flap or free flap reconstruction can be used to fill the orbital defect.

\section{Case Examples}

\section{Case 1: Orbital Intraconal Lesion, Endoscopic Endonasal Approach}

A 71-year-old woman presented with a 2-year history of progressive central vision changes and diplopia. CT revealed a right-sided intraconal orbital mass, medial to the optic nerve (-Fig. 4). The patient underwent endoscopic endonasal approach with 0 and 30 degrees of rigid endoscopes (-Fig. 4). Right ethmoidectomy and maxillary antrostomy were performed. The surgical corridor was developed between the medial and the inferior recti muscles and the lesion was identified, dissected, and resected from the surrounding structures. No reconstruction was performed. The infraorbital neurovascular bundle remained protected. Complete resection of the lesion was achieved as confirmed by postoperative CT scan (-Fig. 4). Postoperatively, the patient developed transient diplopia that resolved within 1 month. There were no surgical complications encountered. Pathology revealed a benign cavernous hemangioma. 

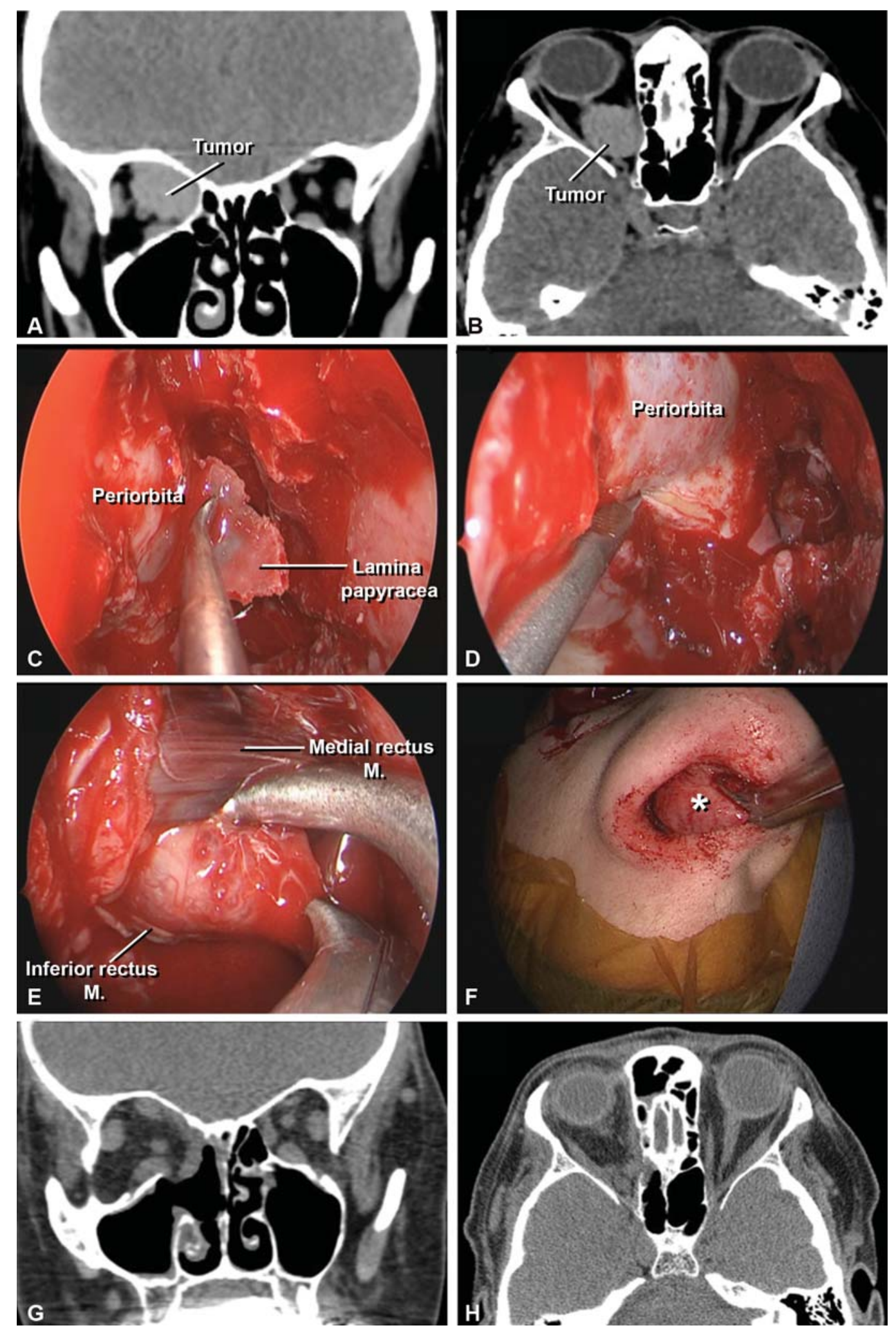

Fig. 4 Case example 1. (A, B) Axial and coronal preoperative noncontrast CT scan demonstrating a well circumscribed right intraconal lesion, with lateral displacement of the optic nerve. (C) 0-degree endoscope; identification and resection of lamina papyracea after maxillary antrostomy, ethmoid, and sphenoidectomy. (D) 0-degree endoscope; incision of the periorbita. (E) 30-degree endoscope; lesion identification, and resection between the inferior and medial rectus with external traction. $(\mathrm{F})$ Lesion $\left(^{*}\right)$ removal. $(\mathrm{G}, \mathrm{H})$ Axial and coronal postoperative noncontrast CT scan demonstrating complete resection of the tumor. CT, computed tomography; $\mathrm{M}$, muscle. 

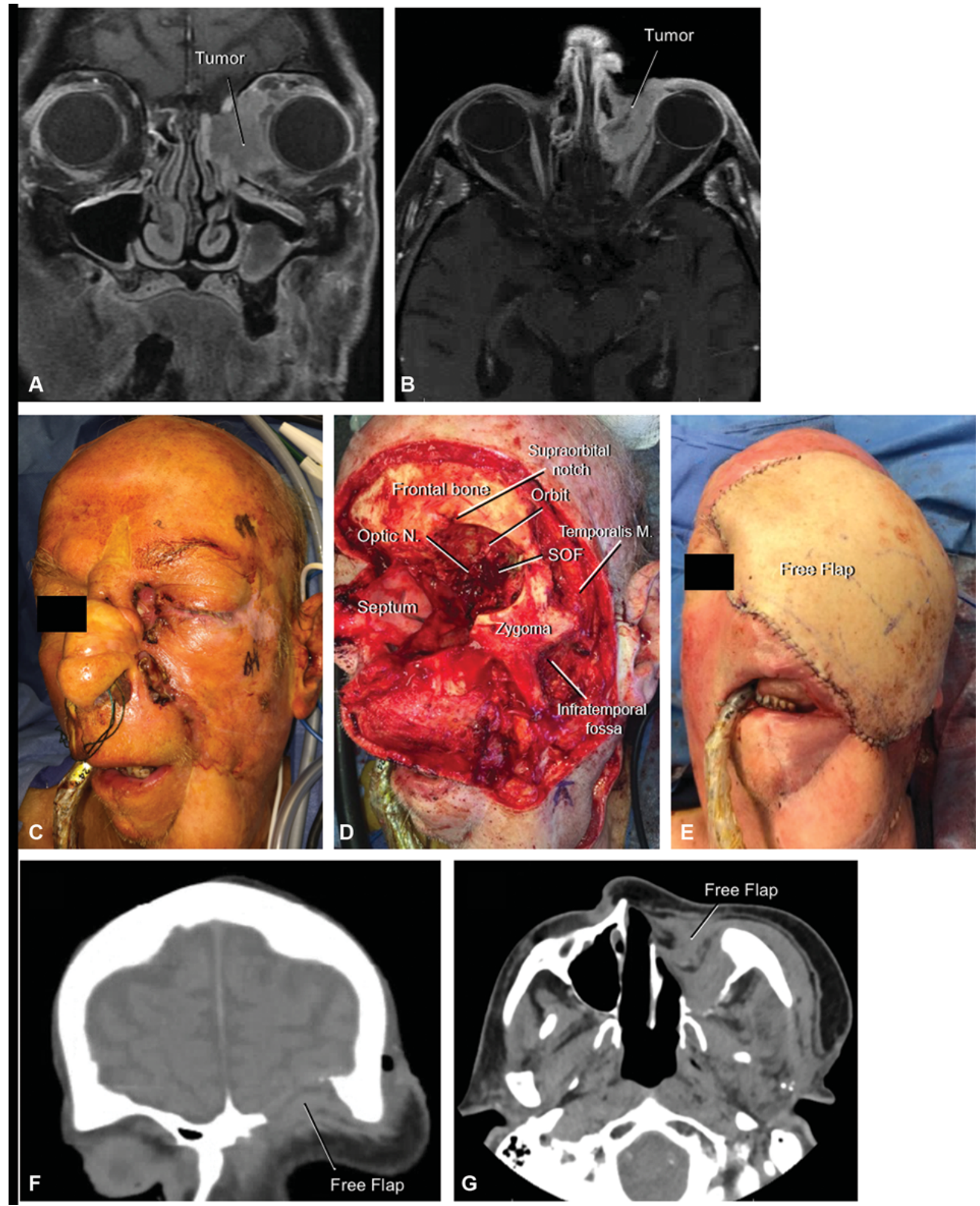

Fig. 5 Case 2, orbital exenteration and reconstruction with vastus lateralis free flap. (A, B) Coronal and axial T1-contrast enhanced MRI demonstrating the tumor extending into the left orbit. (C) Preoperative image of the cutaneous and orbital lesion. (D) Final image after en bloc resection. Note the SOF and optic nerve. (E) Reconstruction with free flap, which was anastomosed to the facial artery. (F, G) Coronal and axial postoperative $\mathrm{CT}$ demonstrating the free flap reconstruction. $\mathrm{CT}$, computed tomography; $\mathrm{N}$, nerve; SOF, superior orbital fissure.

Case 2: Nasal Malignancy with Orbital Extension: Orbital Exenteration and Reconstruction with Free Flap

An 87-year-old male presented with a complex history of T4N0M0 squamous cell carcinoma involving the left mandible. He initially underwent mandibulectomy and left modi- fied radical neck dissection with fibula free flap and postoperative radiation 5 years ago. One year after the mandibulectomy, he presented with cutaneous squamous cell carcinoma involving the nose and underwent MOHS surgery and reconstruction with paramedian forehead flap. 
One year after this resection, the patient presented with a recurrence of the tumor which involved the nasal skin and left cheek. He underwent resection and reconstruction with local advancement flaps followed by postoperative radiation.

The patient was referred to us 1 year after the last surgery with ulcerations due to recurrent tumor involving the nose, left nasolabial sulcus, and left medial canthus. CT and MRI showed extensive tumor involving the left side of the face and orbit. Patient underwent wide en bloc resection including orbital exenteration with negative margins, and reconstruction with vastus lateralis musculocutaneous free flap anastomosed to the facial artery (-Fig. 5 ).

The patient is recovered since 2 years after the radical resection with no evidence of facial recurrence. Unfortunately, a few months ago he presented with new scalp lesions positive for squamous cell carcinoma treated with surgery and a positron emission tomography (PET) scan showed metastatic lung disease and orbital recurrence. The patient is currently under treatment with immunotherapy.

\section{Conflict of Interest}

Grants from NREF, Medtronic, and Zeiss were received during the conduction of the study.

\section{Acknowledgments}

The authors deeply acknowledge the grant support received by the NREF (Neurosurgical Research and Education Foundation), educational grants from Medtronic and Zeiss.

\section{References}

1 Martins C, Costa E Silva IE, Campero A, et al. Microsurgical anatomy of the orbit: the rule of seven. Anat Res Int 2011; 2011:468727

2 Greenfield JP, Anand VK, Kacker A, et al. Endoscopic endonasal transethmoidal transcribriform transfovea ethmoidalis approach to the anterior cranial fossa and skull base. Neurosurgery 2010;66 (05):883-892, discussion 892

3 Niho S, Niho M, Niho K. Decompression of the optic canal by the transethmoidal route and decompression of the superior orbital fissure. Can J Ophthalmol 1970;5(01):22-40

4 Martins C, Yasuda A, Campero A, Ulm AJ, Tanriover N, Rhoton A Jr. Microsurgical anatomy of the dural arteries. Neurosurgery 2005; 56(02):211-251, discussion 211-251

5 Başak S, Karaman CZ, Akdilli A, Mutlu C, Odabaşi O, Erpek G. Evaluation of some important anatomical variations and dangerous areas of the paranasal sinuses by CT for safer endonasal surgery. Rhinology 1998;36(04):162-167

6 Standring S ed. Gray's Anatomy: The Anatomical Basis of Clinical Practice. 40th ed. Edinburgh, Scotland: Elsevier; 2008

7 Peris-Celda MP-NCD, Valentine R, Rhoton AL. Sinonasal and Parasellar anatomy. In: Evans JJ, Kenning TJ, Farrell C., Kshettry V., eds. Endoscopic and Keyhole Cranial Base Surgery. Switzerland: Springer; 2019

8 Stammberger HR, Kennedy DW; Anatomic Terminology Group. Paranasal sinuses:anatomic terminology and nomenclature. Ann Otol Rhinol Laryngol Suppl 1995;167:7-16

9 Stallman JS, Lobo JN, Som PM. The incidence of concha bullosa and its relationship to nasal septal deviation and paranasal sinus disease. AJNR Am J Neuroradiol 2004;25(09):1613-1618

10 Rhoton AL Jr. The orbit. Neurosurgery 2002;51(04):S303-S334
11 Hitotsumatsu T, Rhoton AL Jr. Unilateral upper and lower subtotal maxillectomy approaches to the cranial base: microsurgical anatomy. Neurosurgery 2000;46(06):1416-1452, discussion 1452-1453

12 Schultheiß S, Petridis AK, El Habony R, Maurer P, Scholz M. The transmaxillary endoscopic approach to the orbit. Acta Neurochir (Wien) 2013;155(01):87-97

13 Rhoton AL Jr. The anterior and middle cranial base. Neurosurgery 2002;51(04):S273-S302

14 Lantos JE, Pearlman AN, Gupta A, et al. Protrusion of the infraorbital nerve into the maxillary sinus on $\mathrm{CT}$ : prevalence, proposed grading method, and suggested clinical implications. AJNR Am J Neuroradiol 2016;37(02):349-353

15 Cohen NA, Antunes MB, Morgenstern KE. Prevention and management of lacrimal duct injury. Otolaryngol Clin North Am 2010; 43(04):781-788

16 Rolls ET. The functions of the orbitofrontal cortex. Brain Cogn 2004;55(01):11-29

17 Natori Y, Rhoton AL Jr. Microsurgical anatomy of the superior orbital fissure. Neurosurgery 1995;36(04):762-775

18 Fukuda H, Evins AI, Burrell JC, Iwasaki K, Stieg PE, Bernardo A. The meningo-orbital band: microsurgical anatomy and surgical detachment of the membranous structures through a frontotemporal craniotomy with removal of the anterior clinoid process. J Neurol Surg B Skull Base 2014;75(02):125-132

19 Yasuda A, Campero A, Martins C, Rhoton AL Jr., de Oliveira E, Ribas GC. Microsurgical anatomy and approaches to the cavernous sinus. Neurosurgery 2005;56(01):4-27, discussion 4-27

20 Abed SF, Shams P, Shen S, Adds PJ, Uddin JM, Manisali M. A cadaveric study of the cranio-orbital foramen and its significance in orbital surgery. Plast Reconstr Surg 2012;129(02):307e-311e

21 Turvey TA, Golden BA. Orbital anatomy for the surgeon. Oral Maxillofac Surg Clin North Am 2012;24(04):525-536

22 Dallan I, Castelnuovo P, de Notaris M, et al. Endoscopic endonasal anatomy of superior orbital fissure and orbital apex regions: critical considerations for clinical applications. Eur Arch Otorhinolaryngol 2013;270(05):1643-1649

23 Rhoton AL Jr, Harris FS, Renn WH. Microsurgical anatomy of the sellar region and cavernous sinus. Clin Neurosurg 1977;24:54-85

24 Harris FS, Rhoton AL. Anatomy of the cavernous sinus. A microsurgical study. J Neurosurg 1976;45(02):169-180

25 Rhoton AL Jr. The cavernous sinus, the cavernous venous plexus, and the carotid collar. Neurosurgery 2002;51(04):S375-S410

26 Inoue T, Rhoton AL Jr., Theele D, Barry ME. Surgical approaches to the cavernous sinus: a microsurgical study. Neurosurgery 1990; 26(06):903-932

27 Rhoton AL Jr. The cavernous sinus, the cavernous venous plexus, and the carotid collar. Neurosurgery 2002;51(04):S375-S410

28 Rhoton AL Jr. The sellar region. Neurosurgery 2002;51(04): S335-S374

29 De Battista JC, Zimmer LA, Rodríguez-Vázquez JF, et al. Muller's muscle, no longer vestigial in endoscopic surgery. World Neurosurg 2011;76(3-4):342-346

30 Tong L, Bauer RJ, Buchman SR. A current 10-year retrospective survey of 199 surgically treated orbital floor fractures in a nonurban tertiary care center. Plast Reconstr Surg 2001;108 (03):612-621

31 Robinson TJ, Stranc MF. The anatomy of the medial canthal ligament. Br J Plast Surg 1970;23(01):1-7

32 Mathias RN, Lieber S, de Aguiar PHP, Maldaun MVC, Gardner P, Fernandez-Miranda JC. Interfascial dissection for protection of the nerve branches to the frontalis muscles during supraorbital trans-eyebrow approach: an anatomical study and technical note. J Neurol Surg B Skull Base 2016;77(03):265-270

33 Ostergard TA, Glenn CA, Dekker SE, Bambakidis NC. Is the supraorbital notch a reliable landmark to avoid the frontal sinus? Oper Neurosurg (Hagerstown) 2019;16(03):360-367 
34 Ammirati M, Spallone A, Ma J, Cheatham M, Becker D. An anatomicosurgical study of the temporal branch of the facial nerve. Neurosurgery 1993;33(06):1038-1043, discussion 1044

35 Tellioğlu AT, Tekdemir I, Erdemli EA, Tüccar E, Ulusoy G. Temporoparietal fascia: an anatomic and histologic reinvestigation with new potential clinical applications. Plast Reconstr Surg 2000;105 (01):40-45
36 Spiriev T, Poulsgaard L, Fugleholm K. Techniques for preservation of the frontotemporal branch of facial nerve during orbitozygomatic approaches. J Neurol Surg B Skull Base 2015;76(03):189-194

37 Engle RD, Chaskes M, Wladis E, Pinheiro-Neto CD. Feasibility study for transnasal endoscopic repair of orbital floor fracture with alloplastic implant. Ann Otol Rhinol Laryngol 2016;125(12): 970-975 